\title{
Quality, Conformity, and Conflict: Questioning the Assumptions of Osborn's Brainstorming Technique
}

\author{
Olga Goldenberg' and Jennifer Wiley'
}

Invited

\begin{abstract}
:
Divergent thinking tasks are a popular basis for research on group creative problem solving, or brainstorming. The brainstorming literature has been dominated by research that investigates group performance by measuring the total number of generated ideas using the original rules put forth by Osborn (1953). This review of empirical literature on brainstorming suggests that Osborn was right about many but not all of his intuitions. It discusses the potential advantages of cognitive stimulation, and potential disadvantages of conformity or fixation, due to exposure to others' ideas. Further, although Osborn recommended "withholding criticism," the potential advantages of conflict in interacting problem-solving groups are also discussed. In addition, this review suggests that incorporating performance measures of idea quality, in addition to the ubiquitous measures of idea quantity, can provide useful and unique insights into group brainstorming.
\end{abstract}

Keywords:

brainstorming, idea generation, creative problem solving

${ }^{1}$ University of Illinois at Chicago

The Journal of Problem Solving • volume 3, no. 2 (Winter 2011) 


\section{Introduction}

Historians and philosophers have long wondered about the causes of innovative thinking and problem solving, and have been inquiring into the lives of prominent creators for centuries. One event that had a large impact on modern study of innovation and creativity, particularly from the social-cognitive psychological perspective, was the publication of the first edition of Alex Osborn's book on brainstorming in 1953. Osborn was an advertising firm executive who introduced and developed this technique to help teams generate creative solutions to everyday problems. In the earlier editions of his book, Osborn (1953, 1957) contended that idea generation in teams is far superior to individual brainstorming. In an ideal group brainstorming session described by Osborn, whenever an individual has an idea, he or she verbally announces it to the rest of the team, and a designated person records all of the spoken ideas. The rationale behind this is that team members can benefit from being exposed to the ideas of others. Moreover, Osborn $(1957,1963)$ suggested that the key to the effectiveness of group brainstorming is adherence to certain rules. These rules are: (1) idea quantity is the goal (the more ideas, the better); (2) criticism must be ruled out (evaluation of ideas should be withheld); (3) freewheeling is welcomed (the wilder the idea, the better); and (4) combination and improvement are sought (refine and combine ideas in novel ways). In addition to these basic four rules, Osborn offered a number of other recommendations for effective, successful brainstorming. Some of these assumptions have been empirically tested, and as this review will suggest, Osborn was right about many but not all of his intuitions.

The main goal of the present paper is to review empirical evidence related to the effectiveness of group brainstorming techniques, and to question whether some features and assumptions of the traditional brainstorming approach should be reconsidered. In the first section, we discuss empirical results suggesting that group brainstorming is generally less effective than individual efforts in terms of both quantity and quality. In the second section, we review the various factors that have been considered as explanations for the failure of interacting groups to reach their potential. The third section discusses some approaches that have been able to overcome some of the proposed difficulties and have shown improvements in group performance. The fourth section discusses several additional rules that Osborn also suggested, and questions whether presentation of the full set of four standard rules will lead to the best group performance. A final section reconsiders the traditional features and assumptions involved in the brainstorming technique, and suggests some salient future directions for research.

- volume 3, no. 2 (Winter 2011) 


\section{Is Group Brainstorming Indeed Superior to Individual Brainstorming?}

Osborn (1963) asserted that the key to a successful brainstorming exercise is "quantity, quantity, and more quantity" (p. 129). He based his approach on the assumption that better-quality ideas are produced later rather than earlier in the brainstorming session. His reasoning was that we first need to rid our minds of the common, ordinary ideas before we can uncover or think up more original, creative solutions to a problem. As such, generating a greater number of ideas increases the likelihood that good-quality ideas will be embedded among them (Osborn, 1957, 1963). These intuitions have been later corroborated by empirical evidence of significant positive correlations between the total number of ideas and the number of highly original and practical ideas, although more recent approaches explain this result as more of an artifact of probability in sampling, or an equal-odds rule, such that larger samples of ideas will necessarily contain more creative responses (Diehl \& Stroebe, 1987, 1991; Simonton, 1990).

To test Osborn's $(1953,1957)$ claims about superiority of group brainstorming in a systematic way, Taylor, Berry, and Block (1958) developed and utilized the "nominal group" methodology. In this approach, team performance is compared to the combined efforts of an equal number of individuals working alone. Taylor, Berry, and Block (1958) provided four-person interacting and nominal groups with the four brainstorming rules recommended by Osborn $(1953,1957)$. Next, the researchers instructed both group types to generate creative ideas on three different topics that were similar to the kinds of prompts given in the Torrance Tests of Creative Thinking (TTCT) (how to increase tourism to the United States, advantages and difficulties associated with having an extra thumb on each hand, and how to solve problems the education system faces). The results revealed that interacting groups generated fewer ideas than nominal groups. This is in the opposite direction from Osborn's $(1953,1957)$ predictions.

In addition to quantity, Taylor, Berry, and Block (1958) also measured the quality of the participants' ideas in several different ways, including (1) the number of unique ideas (suggested by only one of the groups involved in the experiment), (2) feasibility of responses, and (3) significance of proposed ideas (as judged by expert raters). Again, results showed that the number of high-quality responses was superior for nominal over interacting groups. Finally, when the total number of ideas was controlled for, the nominal group advantage in quality was eliminated, suggesting that quantity and quality were indeed correlated in this study.

In the years that followed, the nominal group methodology gained popularity among group performance researchers. The majority of later empirical studies of group brainstorming focused on the total number of generated ideas, accepting Osborn's assertion that quantity translates into quality. Typical results were similar to Taylor and colleagues' 
(1958) main finding that interacting groups generated fewer ideas than nominal groups (e.g., Diehl \& Stroebe, 1987; Lamm \& Trommsdorff, 1973). In 1991, Mullen, Johnson, and Salas performed a meta-analysis of 20 brainstorming studies comparing interacting and nominal group brainstorming that converged on the same conclusion. This ubiquitous superiority of nominal groups in terms of the total number of generated ideas relative to their interacting group counterparts has been termed "productivity loss" or "process loss" (Steiner, 1972).

\section{Alternate Measures of Performance on Idea Generation Tasks}

At the same time as Osborn published his brainstorming technique, there was a growth in other psychological research on creative behavior with the development of several test batteries designed to measure creative thinking in individuals and the general population. One of the most widely used such batteries is the TTCT (Torrance, 1962, 1972, 2008). The TTCT sequentially presents test takers with a series of divergent thinking tasks, including an Unusual Uses Test, where individuals are asked to come up with as many original uses for common household objects, such as a knife or paper clip, that no one else would think of. Other prompts include thinking of ways to improve products such as a toy, and considering consequences of scenarios such as being able to walk on air. The responses on these tests have traditionally been scored on fluency (number of responses), originality (number of uncommon responses), flexibility (number of different categories explored), and elaboration (the degree of explanation provided for responses). One reason for the popularity of the TCTT is that it has been shown to predict real-life creative achievement and products in individuals (Plucker, 1999). Other creativity tests use similar idea generation tasks, including Guilford's (1956) Alternate Uses Test, where individuals are asked to come up with as many different uses for a common household object as possible, and the Category Fluency test (Wallach \& Kogan, 1965) that asks individuals to name as many exemplars as possible within a particular category, such as mammals and vegetables.

These tests of divergent thinking, such as the TTCT, suggest several different ways in which the resulting creative idea generation performance of groups can be measured. The most common approach is to measure idea quantity or fluency, as emphasized by Osborn's $(1953,1957,1963)$ call for a large number of solutions, Guilford's (1956) measure of the total number of responses on Alternate Uses tasks, and Wallach and Kogan's (1965) category fluency measure (i.e., the number of instances named within a category). Other approaches attempt to capture aspects of response quality. The most common approach in this respect is attempting to code for the originality of generated ideas as an indicator of creativity. This can be accomplished by using subjective (e.g., ratings of perceived originality) or objective (e.g., novelty determined by the frequency of a given response in a sample) techniques. The TTCT offers normative data for this purpose. Other approaches

- volume 3, no. 2 (Winter 2011) 
might involve considering idea or response diversity by coding the number of different categories explored, or considering the level of detail or embellishment or explanation in responses. Innovative codings that go beyond those used in the TTCT have considered the feasibility, potential effectiveness, and potential significance of ideas as measures of quality, usually established by judgments of trained raters.

As noted above, the typical finding when interacting groups are compared to nominal groups is that the nominal groups produce a larger number of ideas. Perhaps not surprisingly, in general, studies that use quantity-based measures of quality (i.e., the number of unique or highly original ideas) have found nominal group brainstorming to be superior to real group idea generation (Diehl \& Stroebe, 1991; Vroom, Grant, \& Cotton, 1969). However, it is still possible that the interacting groups may be coming up with proportionally more ideas of better quality, but fewer ideas overall. To determine this, researchers often attempt to remove the influence of quantity on their measures by examining metrics such as average quality, the quality of only the best idea or top subset of ideas, or the number of ideas that exceed some high threshold (Diehl \& Stroebe, 1987; Reinig, Briggs, \& Nunamaker, 2007). When brainstorming research studies have considered measures of idea quality, the results offer slightly more evidence for successful group performance (Mullen, Johnson, \& Salas, 1991). When the average quality of responses is considered, typically interacting groups do just as well as nominal groups. For example, while Diehl and Stroebe (1987) found that nominal groups generated a greater number of highly original ideas compared to interacting groups, no significant differences between interacting and nominal groups were found in terms of the average idea originality.

Other measures of idea quality use ratings of idea feasibility (practicality, usefulness, effectiveness) (e.g., Barki \& Pinsonneault, 2001; Diehl \& Stroebe, 1987; Rietzschel, Nijstad, \& Stroebe, 2006, 2007; Ziegler, Diehl, \& Zijlstra, 2000). Again, on these quality measures, interacting groups are no worse than nominal groups in average feasibility of generated ideas (Rietzschel, Nijstad, \& Stroebe, 2006, 2007).

The other measure of idea quality that often shows up in the brainstorming literature is the number of explored semantic categories (also referred to as variety, breadth, and flexibility). The results from these studies suggest that groups in interactive contexts tend to generate ideas from fewer semantic categories compared to nominal groups (Larey \& Paulus, 1999; Ziegler, Diehl, \& Zijlstra, 2000). Nijstad, Stroebe, and Lodewijkx (2002) found that exposure to stimulus ideas from a wide range of semantic categories led to the generation of more diverse ideas compared to no exposure. In contrast, exposure to ideas from a small range of semantic categories led to the generation of more ideas per category. 


\section{Why Is Group Brainstorming Not Effective?}

\section{Social and Motivational Factors}

Researchers have identified a number of factors responsible for the suboptimal performance evidenced in real group brainstorming, with the efforts mostly aimed at explaining the performance differences in quantity rather than quality of generated ideas. Perhaps emphasis on idea quantity is in part due to the fact that there was no clear pattern when interacting and nominal groups were directly compared on idea quality, and in part due to the underlying assumption that solving the productivity loss problem for quantity will indirectly take care of any quality issues as well because the two measures have been shown to correlate highly. The factors that have been argued to be responsible for productivity loss include social, motivational, as well as cognitive processes that are often interrelated.

One of the reasons why groups may fail to realize their potential during idea generation is evaluation apprehension (Camacho \& Paulus, 1995; Diehl \& Stroebe, 1987). Evaluation apprehension essentially results from people's concern of being negatively judged by others. During a typical brainstorming session, individuals orally express their ideas to the group, or they are asked to write them down or type them. With some exceptions (as in the case of electronic brainstorming), this setup makes individual contributions easily identifiable, at least at the moment. Although as part of Osborn's $(1957,1963)$ recommendations, groups are admonished not to criticize each other at this stage of the creative process, members may still feel that they are being evaluated. This identifiability could lead team members to withhold certain potentially good ideas. In contrast, individual brainstormers do not undergo immediate evaluation of ideas, and thus should have fewer evaluation apprehension concerns. Diehl and Stroebe (1987) tested these ideas empirically and found that when participants believed that their individual performance was being evaluated by judges, nominal group performance was impaired more than interacting group performance in terms of idea quantity. However, nominal groups still generated a greater number of ideas than interacting groups. Taking an individual differences approach, Camacho and Paulus (1995) compared ideational productivity of groups and individuals with high versus low social anxiety. The researchers found that teams with high social anxiety members generated fewer creative ideas than teams with low social anxiety members, but no performance differences were observed for the participants who brainstormed alone.

It has been suggested that another reason why group brainstorming is not as productive as individual creative ideation in terms of idea quantity could be due to motivation losses resulting from social loafing, free-riding, and the "sucker" effect. Social loafing occurs when group members feel that no one can find out how much they personally contributed, and thus they may not work as hard as they otherwise would (Karau \& Williams, 1993). 
Diehl and Stroebe (1987) tested this explanation with a brainstorming task. The researchers found that holding group members accountable for their individual contributions did not significantly reduce productivity loss (i.e., did not reduce the difference in the total number of generated ideas between interacting and nominal groups). However, it should be noted that while making individuals accountable for their contributions to the group (increased accountability) decreased social loafing, it could have exacerbated evaluation apprehension.

Similarly, free-riding is a form of motivation loss that results when group members feel that their individual efforts are not crucial to the group product (Kerr \& Bruun, 1983). For example, when a team of four is highly productive, such as when three of the four members are able to contribute a large number of ideas, the fourth group member can reap the benefits of good performance without putting in a lot of effort into generating and/or announcing his or her ideas (Kerr \& Bruun, 1983). In addition, if the other three group members become aware of the fact that others are free-riding on their effort, they can also lose motivation. This is known as the "sucker" effect (Kerr \& Bruun, 1983; Orbell \& Dawes, 1981). Therefore, social loafing, free-riding, and the "sucker" effect can all negatively impact group productivity in brainstorming.

Some researchers have also argued that social comparison processes contribute to the productivity gap between interacting and nominal brainstorming groups in terms of idea quantity (Brown \& Paulus, 1996; Paulus \& Dzindolet, 1993). Specifically, these researchers suggested that on idea generation tasks, individuals tend to match their performance to that of other group members. For example, it is has been documented that interacting group members contribute their ideas at similar rates. In contrast, nominal group participants do not exhibit this tendency (Camacho \& Paulus, 1995; Paulus \& Dzindolet, 1993). Thus, if a low-productivity norm is established early in the brainstorming session, this low level of performance will be maintained over time, and a group's idea generation potential will not be realized (Brown \& Paulus, 1996).

\section{Cognitive Factors}

In addition to the social and motivational factors, Diehl and Stroebe $(1987,1991)$ documented production blocking to be a leading cause of productivity loss in group brainstorming. Production blocking stems from the fact that group members need to take turns speaking during a typical face-to-face brainstorming session. While the group session is in progress, each individual needs to perform three things simultaneously: (1) keep the idea he or she would like to contribute in memory, (2) pay attention to the current speaker to foster cognitive stimulation, and (3) monitor discussion to find an appropriate time to intervene and announce his or her idea. In a series of studies, Diehl and Stroebe (1987, 1991) demonstrated that performing these cognitive tasks simultaneously can lead to 
forgetting the original idea that the person wanted to contribute, and more important, make it difficult to generate additional new ideas during this time. Group members in nominal groups do not have to worry about waiting for a turn to speak because they work individually, and therefore are unaffected by production blocking. Production blocking is thus presumed to have a direct effect on the number of generated ideas.

The conceptualization of groups as information processors suggests another account of productivity loss in group brainstorming (Hinsz, Tindale, \&Vollrath, 1997). Hinsz, Tindale, and Vollrath (1997) proposed that information processing in small, task-performing groups takes place both within the individual team members' minds as well as among them. According to this perspective, group interaction can interfere with information retrieval processes. Thus, in a traditional group brainstorming session, retrieval and announcement of one piece of information by one team member may interfere with another member's retrieval processes. In addition, the presence of other group members may distract individuals from the task, thereby reducing the attentional capacity of group members (Hinsz, Tindale, \& Vollrath, 1997). As a result, these cognitive interference processes put interacting brainstorming groups at a disadvantage relative to their nominal group counterparts, who generate ideas alone, and therefore, are not subject to these effects.

Other researchers have theorized that there are additional cognitive processes that could impair creative group performance on idea generation tasks. Smith (2003) argued that fixation on previously stated ideas can block cognitive operations involved in retrieval, which can affect the quality of group brainstorming. During a group brainstorming session, participants are constantly exposed to others' ideas, which can serve as anchors that may prevent subsequent creative idea generation (Smith, 2003). For example, Smith, Ward, and Schumacher (1993) examined the effect of conformity to examples on creative idea generation by asking their participants to invent new toys and to imagine new life forms. Smith, Ward, and Schumacher (1993) found that participants who were provided with several examples of solutions prior to the idea generation task were more likely to incorporate the exemplified features into their responses compared to participants who were not presented with such exemplars. However, no difference was observed in the total number of generated ideas between the two conditions. These results showed that exposure to others' ideas can affect the type, but not necessarily the number, of creative ideas, demonstrating the importance of including direct measures of both quantity and quality in brainstorming studies.

In a more recent study, Kohn and Smith (2010) examined fixation effects that resulted directly from the ideas suggested by other participants in a brainstorming session. They obtained similar results. Exposure to ideas of others reduced novelty of ideas (as measured by typicality), as well as increased conformity to other participants' ideas (Kohn \& Smith, 2010). These results are consistent with those of other researchers, who also noted 
the convergent tendency of groups in interactive contexts to generate ideas from fewer semantic categories compared to nominal groups (Larey \& Paulus, 1999; Ziegler, Diehl, \& Zijlstra, 2000). Additionally, Kohn and Smith (2010) found no differences in terms of idea quantity. Interestingly, these findings suggest that exposure to the ideas of others does not always produce a positive, stimulating effect, but can sometimes narrow the scope of the idea generation process. Moreover, consistent with previous findings (Smith, Ward, \& Schumacher, 1993), fixation seems to affect the type, not quantity, of ideas. Results in this line of research suggest that it is not always effective to rely on the assumption that idea quantity leads to quality, and that there may be more direct ways to improve idea quality (rather than via idea quantity). A similar line of research by Rietzschel, Nijstad, and Stroebe (2007) attempted to prime brainstormers with a set of subtopics to consider in relation to the problem that was posed. Although introducing subtopics did produce more solutions related to that topic, it did not increase productivity overall. As a result, the priming resulted in less diversity in the generated ideas.

\section{Toward Maximizing Productivity in Group Brainstorming}

\section{Reducing Production Blocking and Evaluation Apprehension}

Despite the negative effects of evaluation apprehension, social loafing, free-riding, "sucker" effects, production blocking, and fixation, group brainstorming remains a popular idea generation technique (Coskun, 2005; Rietzschel, Nijstad, \& Stroebe, 2007). Brainstorming research continues because of the belief that cognitive stimulation resulting from idea sharing is possible if production blocking can be eliminated. Two different models of brainstorming behavior have been developed in recent years to help articulate the idea generation process and the influences on individual and group brainstorming performance (see Brown et al., 1998, and Nijstad \& Stroebe, 2006 for details). Computer simulations of these models are generally consistent with existing empirical findings. Larson (2010) points out that both models predict cognitive stimulation in brainstorming groups, once again suggesting that process gain in brainstorming groups should be possible under the right conditions (such as when production blocking is removed). Consistent with these predictions, Dugosh et al. (2000) found that participants generated a greater number of ideas when listening to a tape recording of stimulus ideas in comparison to interacting. In this experiment, listening to the tape recording exposed participants to others' ideas, while production blocking was eliminated because brainstromers worked alone. Clear benefits of cognitive stimulation were evidenced in the number of responses generated by listeners over those who were not exposed to others' ideas. In another experiment, Dugosh et al. (2000) found that priming individuals with ideas can increase productivity but also can affect the quality of responses. They found that exposing participants to a 
wider variety of stimulus ideas led to improved variety in their responses (similar results were also obtained by Nijstad, Stroebe, \& Lodewijkx, 2002).

One way researchers have attempted to minimize some of the barriers is to capitalize on communication techniques in which group members can communicate with each other concurrently, without the need to coordinate speaking turns (e.g., Dennis \& Valacich, 1993; Valacich, Dennis, \& Nunamaker, 1992). The distracting effects that result from the need to wait for a turn to contribute one's ideas are at the root of production blocking in oral, face-to-face brainstorming. Thus, development of idea-sharing techniques that can overcome this barrier should allow the benefits of cognitive stimulation in group brainstorming to become evident.

One such communication technique can be achieved by utilizing technology. Research studies of electronic brainstorming began to appear in the early 1990s (e.g., Connolly, Jessup, \& Valacich, 1990; Dennis \& Valacich, 1994; Valacich, Dennis, \& Nunamaker, 1992). In a typical electronic brainstorming session, every group member is seated at a private or semiprivate computer terminal and presented with the brainstorming task. Utilizing concurrent communication software, such as the AOL Instant Messenger, participants can type their ideas at any time during the session, while still being able to read ideas of others on the screen. Electronic brainstorming eliminates production blocking, while still allowing for cognitive stimulation effects (Dennis \& Valacich, 1993). This procedure can be adjusted to control the number of ideas visible at a time, as well as the contribution source, allowing one to examine the impact of idea sharing and identifiability on productivity.

In 2005, Dennis and Williams conducted a meta-analysis of the numerous electronic brainstorming studies available at the time to test the effectiveness of the technique. Dennis and Williams (2005) restricted their analysis to the studies that reported the total number of generated ideas. The researchers did find that electronic brainstorming can be used to circumvent productivity losses, but interestingly in these early studies they found the effects depended on group size. Electronic brainstorming in terms of quantity of ideas generated is (a) superior to face-to-face group brainstorming, but only for groups with more than three members, and is (b) superior to nominal group brainstorming, but only for groups with more than nine members. To explain the difference between electronic brainstorming and face-to-face brainstorming, Dennis and Williams (2005) argued that in a small face-to-face group, the benefits associated with reduced production blocking in electronic brainstorming are outweighed by the fact that typing ideas is slower than speaking. This issue is perhaps less acute in today's text-based communication culture. To explain the difference between electronic brainstorming and nominal group brainstorming, Dennis and Williams (2005) suggest that in very large electronic brainstorming groups, the benefits of cognitive stimulation resulting from a large number of shared ideas (or possibly due to the increased feelings of competition) outweigh detrimental effects of process loss. The researchers attribute process loss in electronic brainstorming to slower 
typing speed and performing dual tasks of attending to others' ideas and generating additional, new ideas.

DeRosa, Smith, and Hantula (2007) conducted another meta-analytic review of electronic group brainstorming studies that considered performance measures of idea quality in addition to quantity. The researchers obtained the same results as Dennis and Williams (2005) in terms of the number of generated ideas. In terms of idea quality, DeRosa and colleagues (2007) found that electronic brainstorming groups outperformed face-to-face teams in both the number of high-quality responses and average idea quality. However, electronic brainstorming groups performed as well as nominal groups in the number of high-quality ideas and average idea quality.

In addition to minimizing production blocking, it has been suggested that electronic brainstorming may also help reduce anxiety and evaluation apprehension concerns in groups (e.g., Connolly, Jessup, \& Valacich, 1990; Dennis \& Valacich, 1993). The computer interface can be programmed in a way that the source of individual contributions is kept unknown to the participants. In other words, team members can read all of the group's ideas on the screen, but they do not know who contributed which ideas, with the exception of their own. The available empirical evidence in support of this additional benefit of computer-mediated brainstorming is mixed. For example, Connolly and colleagues (1990) tested the effect of anonymity in four-person groups. In the anonymous group conditions, team members were not introduced to each other prior to the brainstorming session, and there were no identifiers in individual response entries. In contrast, in the identified group conditions, participants briefly met their fellow team members, and their names were included next to each response that they made. Connolly et al. (1990) found that anonymous groups generated a greater number of ideas than identified groups, which was a marginally significant effect. However, other studies with similar manipulations of anonymity found no effect of this variable on the number of generated ideas (Dennis \& Valacich, 1993; Valacich, Dennis, \& Nunamaker, 1992).

A paper-and-pencil alternative to electronic brainstorming is "brainwriting," a procedure in which participants are asked to write their ideas down instead of typing them (VanGundy, 1981). For example, Paulus and Yang (2000) conducted a study that used a version of the brainwriting procedure in which four group members exchanged ideas on cards in a round-robin fashion. Additionally, participants were asked to read and pay attention to the ideas on the cards they received from their neighbors before adding their own ideas. In contrast to electronic brainstorming, this procedure involved face-to-face communication, but still eliminated production blocking by allowing group members to generate ideas continuously without waiting for their turn. Social loafing was also reduced because every group member was given a different color pen to write with, which increased identifiability of individual responses. In addition, the brainwriting procedure might have reduced cognitive interference in idea retrieval processes that might result when others verbally announce their ideas, as suggested by Hinsz, Tindale, and Vollrath (1997). Although 
this brainwriting technique could have also increased evaluation apprehension concerns due to team members' awareness of the source of ideas, Paulus and Yang's (2000) results showed that interacting groups outperformed their nominal counterparts in terms of the total number of generated ideas. This result is one of the only findings in the literature where interacting groups can be seen to outperform a nominal group comparison.

Extending work on brainwriting, Coskun (2005) investigated the quality of performance, in addition to quantity, in interacting and nominal dyads using a procedure that was very similar to that of Paulus and Yang (2000). In contrast to Paulus and Yang (2000), however, the interacting and nominal groups generated the same number of ideas. Thus Coskun was unable to replicate the interacting group advantage, but at least the interacting groups were not worse than the nominal ones. In addition, his results suggested that interacting groups scanned a greater range of semantic categories than nominal groups, although the difference was not statistically significant. Taken together, these results suggest that some aspects of the brainwriting technique may be facilitating group performance to be at least as good as nominal group performance, although unfortunately the advantage of interacting over nominal groups produced by this technique does not seem to be robust across studies (Coskun, 2000, 2005).

\section{Reducing Fixation}

Some researchers have emphasized the importance of incubation, or taking time off from a problem-solving task to allow automatic cognitive processing of stimulus ideas to occur (Smith, 2003; Paulus \& Yang, 2000; Paulus \& Brown, 2003). Osborn (1963) himself suggested that periods of incubation could promote solution insights. Paulus et al. (2006, Experiment 2) found that taking a brief, single break increased the total number of generated ideas in written brainstorming compared to not taking one (although this effect was not observed during an electronic brainstorming session; Experiment 3).

Smith $(1995,2003)$ has suggested that taking brief breaks during a creative problem-solving task can help overcome fixation effects, or mental blocks that result from exposure to examples. It is hypothesized that switching to a new context is likely to lead an individual to take a different initial idea generation path (Smith, 2003). Kohn and Smith (2010, Experiment 3) empirically tested the effects of taking a break on fixation in an electronic brainstorming session. To induce fixation, confederates presented participants with typical ideas at fixed time intervals. After brainstorming for 10 minutes, half of the participants were interrupted and asked to work on an unrelated task for 5 minutes, after which brainstorming was resumed for another 10 minutes. In contrast, participants in the control conditions generated ideas for the same amount of time without interruptions. Kohn and Smith's (2010) results showed that brainstormers in the interrupted condition came up with 86 percent more ideas and explored 57 percent more task-relevant categories than participants who did not take a break. Therefore, breaks helped improve both idea quantity and idea quality (as measured by variety). Moreover, the researchers showed that 
breaks helped to modulate the natural decreasing productivity rates that occur over time in a brainstorming session. Interestingly, these results suggest that taking brief breaks can aid idea generation in a group problem-solving context, but the precise mechanism of underlying incubation effects in both individuals and groups remains largely unknown.

\section{Reconsidering Osborn's Additional Rules}

Many brainstorming studies in the first several decades after the publication of Osborn's $(1957,1963)$ book relied only on the four original rules he recommended. However, in addition to the basic set, Osborn suggested a number of other recommendations for optimizing the effectiveness of a brainstorming session, such as including incubation periods, using a group size of a dozen, using facilitators and session leaders, providing encouragement, supplementing group discussion with individual brainstorming, introducing idea-spurring questions, increasing concentration and effort, introducing diversity to the group, taking notes, making checklists, and setting deadlines. As already discussed, several of these corollaries seem to be consistent with some recent findings-such as the advantages found in larger groups and from incubation periods. In recent years, researchers have begun to explore and empirically test some more of these "forgotten" intuitions (Paulus \& Brown, 2003).

\section{Facilitator Studies}

Offner, Kramer, and Winter (1996) investigated how the presence of a facilitator trained in participation encouragement and reinforcement of the brainstorming rules affects ideational productivity in interactive teams. Specifically, the facilitators were charged with keeping the group moving by making sure team members did not violate the four brainstorming rules suggested by Osborn, encouraging quiet members to participate, and making sure the participants stayed focused on the brainstorming task and avoided off-topic discussions. They also summarized previously suggested ideas. Offner, Kramer, and Winter (1996) found that groups with a facilitator generated more ideas than groups without a facilitator and the same number of ideas as nominal groups. However, because the researchers did not include measures of idea quality, it remains unknown how facilitator behaviors might have affected this aspect of performance. For instance, one might speculate that although minimizing off-topic discussions and summarizing could lead to generation of a greater number of ideas, it can also exacerbate fixation effects. As Kohn and Smith (2010) demonstrated, brief interruptions help to reduce conformity and increase the variety of generated responses. Thus, preventing distractions could adversely affect the quality of performance.

In a similar study, Oxley, Dzindolet, and Paulus (1996) compared the effectiveness of trained, highly trained, or untrained facilitators in group brainstorming. In the trained 
facilitator condition, a graduate student helped guide group discussions by interrupting when participants diverged from the task, calling upon nonparticipating members, reminding brainstormers not to criticize ideas, and repeating the brainstorming topic if there was a long pause. Facilitators in the highly trained facilitator condition practiced leading sessions for additional several hours. Oxley, Dzindolet, and Paulus (1996) found that groups with a highly trained facilitator generated (a) as many ideas as nominal groups and (b) more ideas than interacting groups with trained and untrained facilitators. However, just as in the Offner, Kramer, and Winter (1996) study, quality was not assessed. It seems possible that some of the acts of the facilitators, such as minimizing off-task divergence and repeating the topic during silences, could have reduced the originality of generated ideas.

\section{Brainstormer Training}

The above findings suggest that the presence of facilitators who reinforce the rules in interactive brainstorming can be beneficial, at least in terms of idea quantity. However, in a more recent study, Paulus et al. (2006, Experiment 1) found that the presence of an active facilitator (who reminded participants about the rules and encouraged them to explore previous categories) did not impact the quantity of generated ideas compared to a condition where participants were given a set of additional brainstorming rules that they could enforce themselves. Specifically, in some of the experimental conditions, in addition to the four original brainstorming rules, four-person interacting and nominal groups were instructed to stay focused on the task, keep the session going, encourage others to contribute, avoid criticism, and return to previously explored categories. The main dependent variable in this experiment was again the total number of non-redundant ideas. Idea quantity in the active facilitator conditions did not exceed quantity in additional rules conditions; however, groups in the additional rules conditions generated significantly more ideas than groups in the standard brainstorming rules conditions. These results seemed to be due to higher levels of efficiency (using fewer words to express ideas) in the extra-rule groups. It is not clear why these extra instructions promoted shorter but more numerous solutions. Moreover, Paulus and colleagues (2006) also obtained the typical finding of nominal groups outperforming interacting ones. Therefore, these results suggest that the additional rules provided by facilitators, and not the mere presence of facilitators, may improve productivity in brainstorming groups. But even this improvement does not eliminate the typical performance gap between interacting and nominal brainstorming in terms of idea quantity. As in the other above-mentioned experiments that manipulated the administration of additional group brainstorming rules, we are curious about how these additional instructions might affect idea quality. For example, instructing participants to return to previously explored categories could increase ideational fluency, but it might limit the variety of ideas.

- volume 3, no. 2 (Winter 2011) 
Other approaches undertaken to improve brainstorming performance include goal setting and training brainstormers how to be more productive, both of which have been recommended by Osborn $(1957,1963)$ as well. For example, Litchfield $(2009)$ found that supplementing traditional brainstorming rules with specific, difficult quantity goals increased the number of generated ideas among individuals compared to the brainstorming rules or vague quantity goals presented alone. Paulus and Dzindolet (1993) found that providing goals for a "typical" quantity of ideas also improved the performance of groups in terms of number of ideas. However, neither of these goal-setting studies investigated effects on measures of quality.

In a different study, Baruah and Paulus (2008) investigated whether educating group members about successful brainstorming behaviors would improve the quantity and quality of team performance beyond simple practice effects. In the trained conditions, groups of three or four were provided with the following tips prior to the face-to-face brainstorming session: (a) generate as many ideas as possible related to each group member's area of expertise, major, and ethnicity, (b) do not tell stories or explain ideas, (c) listen to others and build upon their ideas, and (d) link different features and characteristics of ideas. In addition to these tips, groups were also reminded that individual contributions to the group were important and would be evaluated, and were provided with feedback about their performance in a practice session. This extensive training increased not only the number but also the average originality of generated ideas compared to groups that spent the same amount of time in practice sessions. Although this is an exciting finding of an increase in quality, since all tips were presented jointly during training, it is difficult to assess their independent effects on performance.

\section{Supplementing Group Discussion with Individual Brainstorming}

Some recent studies provide some support for the suggestion originally proposed by Osborn (1963) that interleaving individual and group attempts may be beneficial. Dugosh et al.'s (2000) results suggest that exposure to initial ideas can improve later performance. Nemeth et al. (2004) also report a result where the quality of an initial group interaction led to better individual productivity following the session. Incubation effects may also be interpreted as providing an opportunity to interleave group and individual efforts.

\section{Concentration and Effort}

Some studies have manipulated the amount of attention that individuals need to pay to others' suggestions by providing memorization instructions. In Dugosh et al. (2000), this improved performance among interacting groups (although the effect was not replicated in Paulus \& Yang, 2000). These results are interpreted as part of stimulation effects-that others' ideas cannot stimulate memory unless they are attended to. And, on a related note, although studies have not explored the role of priming groups with spurring questions per 
se, as reviewed above, there has been work showing the effects of priming brainstormers with a set of ideas or a set of subtopics to consider.

\section{Alternatives to Osborn's Brainstorming Rules: The Importance of Dissent}

Although the empirical results discussed above provide support for some of Osborn's $(1957,1963)$ intuitions about effective group brainstorming, his assumptions about the benefits of withholding criticism have been challenged. Several studies are now suggesting that one way to bring group performance to the same, or even higher, level as nominal groups is to actually harness the potential of dissent (Connolly, Jessup, \& Valacich, 1990; Nemeth \& Nemeth-Brown, 2003; Smith, 2008).

Specifically, Nemeth and Nemeth-Brown (2003) proposed that the poor performance of interacting groups relative to their nominal counterparts can also be, in part, explained by the tendency of majorities to stimulate convergent thought. Nemeth and Kwan (1985) empirically demonstrated that majorities can stimulate less original and more conventional thinking. In their study, after individuals were shown a series of blue slides, participants in the experimental condition were exposed to a majority who called the slides green, while participants in the control condition were not exposed to such a majority. In another condition, however, participants were exposed to a minority, rather than a majority, who called the slides green. Nemeth and Kwan (1985) found that this manipulation affected the quality of responses on a subsequent creativity task. Specifically, individuals exposed to the opinion of the majority, despite its inaccuracy, generated more conventional responses on a word association task than did participants in the control condition. In contrast, participants exposed to the dissenting minority generated more original word associations than those in the control. Thus, the researchers were able to show that the tendency of majorities to stimulate conventional thinking can be overcome by dissenting minority (persistence in a position that differs from a unanimous majority).

There is also evidence that presence of a genuine dissenting minority can help improve performance on an idea generation task. Nemeth, Brown, and Rogers (2001) presented four-person groups with a vacation-scheduling task, in which participants were asked to generate a list of potential solution alternatives before selecting one of three candidates. In the "devil's advocate" conditions, all participants were explicitly told about the role of one of the team members to serve as a "devil's advocate," while in the authentic dissent condition, dissent occurred naturally in the group. Nemeth, Brown, and Rogers (2001) also included a control condition, in which all group members favored the same candidate. One of the dependent variables was a composite score of the number of ideas and average quality of a solution set generated by each group. The results revealed that groups in the authentic minority condition outperformed those in the "devil's advocate" 
and control conditions in both quantity and overall quality of solutions. The Nemeth, Brown, and Rogers (2001) study highlights the value of genuine dissent in brainstorming; however, group performance was not compared to nominal controls.

In another study, Nemeth and colleagues (2004) compared the effectiveness of brainstorming rules that have been traditionally used in research, including the rule to withhold criticism (one of Osborn's [1957, 1963] tenets), with the opposite rule: encourage debate and criticism. Nemeth et al. (2004) randomly assigned five-person groups, with one person serving as a recorder, to one of three conditions. In the traditional brainstorming condition, participants were asked to generate as many solutions as possible to the problem of traffic congestion in their city. In addition, they were instructed that freewheeling was welcomed, and most important, criticism should be ruled out. In the debate condition, the same rules were provided, except that groups were encouraged to debate and criticize each other. The third condition served as a control with no additional rules other than a quantity goal. The total number of ideas served as the measure of performance. The group brainstorming session was followed by an individual session during which participants wrote down ideas they had not expressed during the group session and any additional ideas they had at the moment. Although there was no difference among conditions in productivity during the group phase (which is the usual measure in the literature), Nemeth et al. (2004) found that total productivity (combined performance during the group and individual sessions) in the debate condition was higher than both the control and traditional brainstorming condition. Total productivity in the traditional brainstorming condition did not differ significantly from the control.

Finally, Smith (2008) explored the benefits of dissent on creative idea generation in a dyad paradigm, where some dissenting dyads were informed that their positions represented minority and majority opinions on the topic. The performance of these labeled groups was compared to other dissenting dyads and agreeing dyads. In this study, the dyads who were informed that they represented minority and majority positions generated ideas from a broader range of semantic categories. This suggests that dissent may have positive effects on the quality of idea generation.

The "withhold evaluation" rule is not the only one that might be questioned. Given concerns about fixation from the ideas of others, the "build on" goal may also be problematic, especially for quality outcomes. And if the goal is really quality and not quantity, one wonders what would happen if the emphasis on quality was made explicit.

\section{Conclusion and Implications}

Idea generation and divergent thinking are core components of the creative problemsolving process. Since the publication of the first edition of Osborn's book in 1953, there has been a significant amount of research on group brainstorming. Empirical tests generally 
support many of Osborn's intuitions. For example, it seems that Osborn was correct in his recommendations that brainstormers should take breaks during a brainstorming session. Brainstorming seems to become more productive via the use of trained facilitators to lead group discussions. Brainstorming also appears to become more effective when specific goals and quotas for brainstorming sessions are set.

However, several other assumptions about the effectiveness of brainstorming have not been supported. Most critically, there has not been much evidence in favor of Osborn's $(1953,1957)$ belief about the superiority of group brainstorming over individual creative idea generation. However, as Paulus and Brown (2003) point out, in the third edition of his book, Osborn (1963) revised his original claim and suggested that group collaboration is effective only as a supplement to individual brainstorming. Moreover, even though almost every single brainstorming study suggests that nominal groups outperform interacting groups, there is still hope that cognitive stimulation is possible under the right conditions. There appears to be an interesting tension between stimulation from others' ideas and fixation due to that exposure. These cognitive ramifications of group problem-solving interactions have only begun to be explored, but are critical for discovering the conditions that may allow for the potential of brainstorming groups to be realized.

Another observation contrary to Osborn's original set of rules has emerged from recent findings. It suggests that there may be an important role for dissent and evaluation in creative idea generation. This is contrary to Osborn's $(1973,1957)$ admonitions against criticism of ideas during the generation process. Clearly, there is a balance to be struck between individuals' experiencing a fear of participating and the benefits of accountability and constructive criticism on group products.

Another theme that emerged from this review of studies in the group brainstorming literature is that manipulations of social and situational variables aimed at increasing the number of creative ideas in interactive groups may not always improve idea quality. It is argued that there may be more direct ways to increase originality and variety of ideas rather than via quantity. Therefore, it is important that future research on brainstorming includes assessment of idea quality in addition to quantity to gain more insight into how each of these performance indicators is affected by any variable or manipulation that is in question. Measuring the quality of responses in creative idea generation research offers the potential to learn something above and beyond what we can gauge with sole measures of idea quantity.

Another area of recent attention is, instead of examining idea generation as a task in isolation, putting it back in the context of innovation and organizations. A number of creativity researchers argued that the generation of a large number of ideas that is achieved by the brainstorming technique is one of many stages in the creative process. Idea generation is an initial phase that may follow problem identification, but in order for innovation to occur, it must be followed by phases of idea evaluation, selection, and implementation 
(Nijstad, Diehl, \& Stroebe, 2003; Nijstad \& Levine, 2007; West, 1990, 2002). Although this progression was also recognized by Osborn (1963), until recently much less attention in research has been paid to these later processing stages (Blair \& Mumford, 2007; Putman \& Paulus, 2009; Rietzschel, Nijstad, \& Stroebe, 2006). This work is just beginning and more research is needed in this area.

This new direction has obvious implications for the innovation implementation in organizational settings. Supporting West's $(1990,2002)$ arguments, generating a large quantity of ideas, even if they are of good quality, is not sufficient for successful implementation.

A final point about context is that for the most part studies of brainstorming have been done using ad hoc groups of undergraduate participants solving problems that most typically involve thinking of uses for a paperclip or considering improvements to their campus. More systematic work is needed in actual organizational settings for a wide variety of reasons. Only once careful research is done in authentic organizational contexts can we understand the potential of brainstorming for spurring innovation, as well as how the broader climate or culture of an organization may matter (Heslin, 2009; West, 2002). One intriguing possibility is that advantages of group brainstorming may only be realized in real-world contexts that have not yet been carefully studied, or they may be found in ancillary benefits (i.e., enjoyment, organizational memory, and supporting a particular corporate climate) rather than in measures of the quantity or quality of ideas per se.

\section{References}

Barki, H., \& Pinsonneault, A. (2001). Small group brainstorming and idea quality: Is electronic brainstorming the most effective approach? Small Group Research, 32, 158-205.

Baruah, J., \& Paulus, P. B. (2008). Effects of training on idea generation in groups. Small Group Research, 39, 523-541.

Blair, C. S., \& Mumford, M. D. (2007). Errors in idea evaluation: Preference for the unoriginal? Journal of Creative Behavior, 41, 197-222.

Brown, V. R., \& Paulus, P. B. (1996). A simple dynamic model of social factors in group brainstorming. Small Group Research, 27, 91-114.

Brown, V., Tumeo, M., Larey, T. S., \& Paulus, P. B. (1998). Modeling cognitive interaction during brainstorming. Small Groups Research, 29, 495-526.

Camacho, L. M., \& Paulus, P. B. (1995). The role of social anxiousness in group brainstorming. Journal of Personality and Social Psychology, 68, 1071-1080.

Connolly, T., Jessup, L. M., \&Valacich, J. S. (1990). Effects of anonymity and evaluative tone on idea generation in computer-mediated groups. Management Science, 36, 689-703.

Coskun, H. (2000). The effects of out-group comparison, social context, intrinsic motivation, and collective identity in brainstorming groups. Unpublished doctoral dissertation, University of Texas, Arlington. 
Coskun, H. (2005). Cognitive stimulation with convergent and divergent thinking exercises in brainwriting: Sequence priming and group context. Small Group Research, 36, 466-498.

Dennis, A. R., \& Valacich, J. S. (1993). Computer brainstorms: More heads are better than one. Journal of Applied Psychology, 78, 531-537.

Dennis, A. R., \& Valacich, J. S. (1994). Group, sub-group, and nominal idea generation: New rules for a new media? Journal of Management, 20, 723-736.

Dennis, A. R., \& Williams, M. L. (2005). A meta-analysis of group size effects in electronic brainstorming: More heads are better than one. International Journal of e-Collaboration, 1, 24-42.

DeRosa, D. M., Smith, C. L., \& Hantula, D. A. (2007). The medium matters: Mining the long-promised merit of group interaction in creative idea generation tasks in a meta-analysis of the electronic group brainstorming literature. Computers in Human Behavior, 23, 1549-1581.

Diehl, M., \& Stroebe, W. (1987). Productivity loss in brainstorming groups: Toward the solution of a riddle. Journal of Personality and Social Psychology, 53, 497-509.

Diehl, M., \& Strobe, W. (1991). Productivity loss in idea-generating groups: Tracking down the blocking effect. Journal of Personality and Social Psychology, 61, 392-403.

Dugosh, K. L., Paulus, P. B., Roland, E. J., \& Yang, H.-C. (2000). Cognitive stimulation in brainstorming. Journal of Personality and Social Psychology, 79, 722-735.

Guilford, J. P. (1956). The structure of intellect. Psychological Bulletin, 53, 367-293.

Heslin, P. A. (2009). Better than brainstorming? Potential contextual boundary conditions to brainwriting for idea generations in organizations. Journal of Occupational and Organizational Psychology, 82, 129-145.

Hinsz, V. B., Tindale, R. S., \& Vollrath, D. A. (1997). The emerging conceptualization of groups as information processors. Psychological Bulletin, 121, 43-64.

Karau, S. J., \& Williams, K. D. (1993). Social loafing: A meta-analytic review and theoretical integration. Journal of Personality and Social Psychology, 65, 681-706.

Kerr, N., \& Brunn, S. (1983). Dispensability of member effort and group motivation losses: Free rider effects. Journal of Personality and Social Psychology, 44, 78-94.

Kohn, N. W., \& Smith, S. M. (2010). Collaborative fixation: Effects of others' ideas on brainstorming. Applied Cognitive Psychology. doi: 10.1002/acp. 1699.

Lamm, H., \& Trommsdorff, G. (1973). Group versus individual performance on a task requiring ideation proficiency (brainstorming): A review. European Journal of Social Psychology, 3, 362-388.

Larry, T. S., \& Paulus, P. B. (1999). Group preference and convergent tendencies in small groups: A content analysis of group brainstorming performance. Creativity Research Journal, 12, 175-184.

Larson, J. R., Jr. (2010). In Search of Synergy in Small Group Performance. Psychology Press.

- volume 3, no. 2 (Winter 2011) 
Litchfield, R. C. (2009). Brainstorming rules as assigned goals: Does brainstorming really improve idea quantity? Motivation and Emotion, 33, 25-31.

Mullen, B., Johnson, C., \& Salas, E. (1991). Productivity loss in brainstorming groups: A meta-analytic integration. Basic and Applied Social Psychology, 12, 332-340.

Nemeth, C., Brown, K., \& Rogers, J. (2001). Devil's advocate versus authentic dissent: Stimulating quantity and quality. Journal of Social Psychology, 31, 707-720.

Nemeth, C., \& Kwan, J. (1985). Originality of word associations as a function of majority vs. minority influence processes. Social Psychology Quarterly, 48, 277-282.

Nemeth, C. J., \& Nemeth-Brown, B. (2003). Better than individuals? The potential benefits of dissent and diversity for group creativity. In P. B. Paulus \& B. A. Nijstad (Eds.), Group Creativity: Innovation through Collaboration (pp.63-84). Oxford University Press.

Nemeth, C. J., Personnaz, B., Personnaz, M., \& Goncalo, J. A. (2004). The liberating role of conflict in group creativity: A study in two countries. European Journal of Social Psychology, 34, 365-374.

Nijstad, B. A., Diehl, M., \& Stroebe, W. (2003). Cognitive stimulation and interference in idea-generating groups. In P. B. Paulus \& B. A. Nijstad (Eds.), Group Creativity: Innovation through Collaboration (pp. 137-159). Oxford University Press.

Nijstad, B. A., \& Levine, J. M. (2007). Group creativity and the stages of creative problem solving. In M. Hewstone, H. A. W. Schut, J. B. F. De Wit, K. Van Den Bos, \& M. S. Stroebe (Eds.), The Scope of Social Psychology: Theory and Applications (pp. 159-171). Psychology Press.

Nijstad, B. A., \& Stroebe, W. (2006). How the group affects the mind: A cognitive model of idea generation in groups. Personality and Social Psychology Review, 10, 186-213.

Nijstad, B. A., Stroebe, W., \& Lodewijkx, H. F. M. (2002). Cognitive stimulation and interference in groups: Exposure effects in an idea generation task. Journal of Experimental Social Psychology, 38, 535-544.

Offner, A. K., Kramer, T. J., \& Winter, J. P. (1996). The effects of facilitation, recording, and pauses on group brainstorming. Small Group Research, 27, 283-298.

Orbell, J., \& Dawes, R. (1981). Social dilemmas. In G. Stephenson \& J. H. Davis (Eds.), Progress in Applied Social Psychology (Vol. 1). Wiley.

Osborn, A. F. (1953). Applied Imagination: Principles and Procedures of Creative Thinking. Scribners.

Osborn, A. F. (1957). Applied Imagination: Principles and Procedures of Creative Problemsolving (2nd ed.). Scribners.

Osborn, A. F. (1963). Applied Imagination: Principles and Procedures of Creative Problemsolving (3rd rev. ed.). Scribners.

Oxley, N. L., Dzindolet, M. T., \& Paulus, P. B. (1996). The effects of facilitators on the performance of brainstorming groups. Journal of Social Behavior and Personality, 11, 633-646. 
Paulus, P. B., \& Brown, V. R. (2003). Enhancing ideational creativity in groups: Lessons from research on brainstorming. In P. B. Paulus \& B. A. Nijstad (Eds.), Group Creativity: Innovation through Collaboration (pp. 110-136). Oxford University Press.

Paulus, P. B., \& Dzindolet, M. T. (1993). Social influence processes in group brainstorming. Journal of Personality and Social Psychology, 64, 575-262.

Paulus, P. B., Nakui, T., Putman, V. L., \& Brown, V. R. (2006). Effects of task instructions and brief breaks on brainstorming. Group Dynamics: Theory, Research, and Practice, 10, 206-219.

Paulus, P. B., \& Yang, H.-C. (2000). Idea generation in groups: A basis for creativity in organizations. Organizational Behavior and Human Decision Processes, 82, 76-87.

Plucker, J. A. (1999). Is the proof in the pudding? Reanalyses of Torrance's (1958 to present) longitudinal data. Creativity Research Journal, 12, 103-114.

Putman, V. L., \& Paulus, P. B. (2009). Brainstorming, brainstorming rules, and decision making. Journal of Creative Behavior, 43, 23-39.

Reinig, B. A., Briggs, R. O., \& Nunamaker, J. F., Jr. (2007). On the measurement of ideation quality. Journal of Management Information Systems, 23, 143-161.

Rietzschel, E. F., Nijstad, B. A., \& Stroebe, W. (2006). Productivity is not enough: A comparison of interactive and nominal brainstorming on idea generation and selection. Journal of Experimental Social Psychology, 42, 244-251.

Rietzschel, E. F., Nijstad, B. A., \& Stroebe, W. (2007). Relative accessibility of domain knowledge: The effects of knowledge activation on the quantity and originality of generated ideas. Journal of Experimental Social Psychology, 43, 933-946.

Simonton, D. K. (1990). Psychology, Science and History: An Introduction to Historiometry. Yale University Press.

Smith, S. M. (1995). Fixation, incubation, and insight in memory, problem solving, and creativity. In S. M. Smith, T. B. Ward, \& R. A. Finke (Eds.), The Creative Cognition Approach (pp. 135-155). MIT Press.

Smith, S. M. (2003). The constraining effects of initial ideas. In P. B. Paulus \& B. A. Nijstad (Eds.), Group Creativity: Innovation through Collaboration (pp. 15-31). Oxford University Press.

Smith, S. M. (2008). Adding minority status to a source of conflict: An examination of influence processes and product quality in dyads. European Journal of Social Psychology, 38, 75-83.

Smith, S. M., Ward, T. B., \& Schumacher, J. S. (1993). Constraining effects of examples in a creative generation task. Memory \& Cognition, 21, 837-845.

Steiner, I. D. (1972). Group Process and Productivity. Academic Press.

Taylor, D. W., Berry, P. C., \& Block, C. H. (1958). Does group participation when brainstorming facilitate or inhibit creative thinking? Administrative Science Quarterly, 3, 23-47. 
Torrance, E. P. (1962). Guilding Creative Talent. Prentice Hall.

Torrance, E. P. (1972). Predictive validity of the Torrance Tests of Creative Thinking. Journal of Creative Behavior, 6, 236-252.

Torrance, E. P. (2008). Torrance Tests of Creative Thinking: Norms-technical manual, verbal forms $A$ and $B$. Scholastic Testing Service.

Valacich, J. S., Dennis, A. R., \& Nunamaker, J. F., Jr. (1992). Group size and anonymity effects on computer-mediated idea generation. Small Group Research, 23, 49-73.

VanGundy, A. B. (1981). Techniques for Structured Problem Solving. Van Nostrand Reinhold.

Vroom, V. H., Grant, L. D., \& Cotton, T. W. (1969). The consequences of social interaction in group problem-solving. Organizational Behavior and Human Performance, 4, 77-95.

Wallach, M. A., \& Kogan, N. (1965). Models of Thinking in Young Children. Holt, Rinehart, and Winston.

West, M. A. (1990). The social psychology of innovation in groups. In M. A. West \& J. L. Farr (Eds.), Innovation and Creativity at Work: Psychological and Organizational Strategies (pp. 309-333). Wiley.

West, M. A. (2002). Ideas are ten a penny: It's team implementation not idea generation that counts. Applied Psychology: An International Review, 51, 411-424.

Ziegler, R., Diehl, M., \& Zijlstra, G. (2000). Idea production in nominal and virtual groups: Does computer-mediated communication improve group brainstorming? Group Processes and Intergroup Relations, 3, 141-158.

Paper submitted: October 5, 2010

Paper accepted: December 11, 2010 